\title{
Power-Law-Distributed Dark States are the Main Pathway for Photobleaching of Single Organic Molecules
}

\author{
Jacob P. Hoogenboom, ${ }^{*}$ Erik M. H. P. van Dijk, Jordi Hernando, Niek F. van Hulst, and María F. García-Parajó \\ Applied Optics group, Faculty of Science \& Technology and MESA ${ }^{+}$Institute for Nanotechnology, University of Twente, \\ P.O. Box 217, NL-7500AE Enschede, the Netherlands
}

(Received 7 February 2005; published 26 August 2005)

\begin{abstract}
We exploit the strong excitonic coupling in a superradiant trimer molecule to distinguish between longlived collective dark states and photobleaching events. The population and depopulation kinetics of the dark states in a single molecule follow power-law statistics over 5 orders of magnitude in time. This result is consistent with the formation of a radical unit via electron tunneling to a time-varying distribution of trapping sites in the surrounding polymer matrix. We furthermore demonstrate that this radicalization process forms the dominant pathway for molecular photobleaching.
\end{abstract}

PACS numbers: 78.67. $-\mathrm{n}, 33.50 . \mathrm{Hv}, 34.70 .+\mathrm{e}, 71.35 .-\mathrm{y}$

The fluorescence signal of single quantum emitters is characterized by the random succession of bright (emitting) and dark (nonemitting) periods. This random telegraph signal results from excursions to levels from which the excited electron returns to the ground state via a nonfluorescent pathway [1]. One well-studied cause of on-off intermittency in single fluorophores is intersystem crossing from the singlet excited state to the triplet state [2-5]. Besides fast triplet blinking, particularly long-lived dark (LD) states with typical off times $\tau_{\text {off }} \gg \tau_{\text {triplet }}$ have been reported [4-8]. Such LD states have been extensively studied on semiconductor nanocrystals [9-13], yet only few studies on other types of single emitters have been reported $[4,6,14]$. In the case of fluorescent organic molecules, the LD states are discussed in terms of the reversible formation of nonfluorescent photo-oxidation products $[7,15]$, and the formation of radical anions or cations via intersystem crossing [14] or electron tunneling processes [4]. So far, however, data on LD state dynamics in single molecules have been hard to acquire.

Aside from temporary loss of emission, single-molecule studies also suffer from photobleaching, a photo-induced reaction, usually oxidation [7], yielding a nonfluorescent product. In particular the triplet and higher excited states have been considered as important intermediate states in photobleaching [16]. Although little is known about the nature of this reaction and the pathway involved, it appears that oxygen plays an important role [15,17], either being the primary oxidation agent $[7,16]$, or assisting in the population and depopulation of other excited states than the singlet [16]. A complex pathway involving multiple intermediate states was suggested by $\mathrm{Ha}$ et al. [17]. Recently, it was also shown that radical dark states might be involved in the photobleaching pathway [16]. Singlemolecule fluorescence studies, however, cannot unambiguously distinguish between a (temporary) excursion to a LD state longer than the observation time and a (permanent) photobleaching event.
Here, we exploit the stepwise photobleaching process of a system consisting of three strongly coupled fluorophores to distinguish between collective LD states and photobleaching, which leads to a permanent change in emission properties. We demonstrate that, on a single-molecular level, the population and depopulation kinetics of the LD state follow power-law statistics over 5 orders of magnitude in time, indicative of radical formation via an electron tunneling process. We directly disentangle the intimate relationship between the intermediate radical dark state and the photodegradation pathway, proving that photoinduced radicalization through electron tunneling forms the main pathway for photobleaching.

The system under investigation is a perylene trimer (TPD) that consists of three rigidly linked tetraphenoxyperylene diimide (PD) units (inset in Fig. 1). The conjugation paths of the PD units are perpendicular to each other separating the units electronically. However, their transition dipoles are arranged in a head-to-tail configuration and are strongly coupled by dipolar interaction [18]. We have recently demonstrated that this strong coupling leads to exciton delocalization at room temperature as evidenced by a reduction of the fluorescence lifetime (superradiance) and by a redshift and reduction of vibronic features in the emission spectrum [18].

A home-built confocal microscope was used to measure single-molecule fluorescence intensity, lifetime $\left(\tau_{f}\right)$, and dipole orientation [8]. Samples were prepared by spincoating a solution of $10^{-9} \mathrm{M}$ of TPD and $5 \mathrm{~g} / 1$ poly(methylmethacrylate) (PMMA) in toluene on an $\mathrm{O}_{2}$-plasma etched glass cover slide. Molecules were excited at $\lambda=568 \mathrm{~nm}$ with circularly polarized pulses of $280 \mathrm{fs}$ duration, $8 \mathrm{MHz}$ repetition rate and an average power density of $2.5 \mathrm{~kW} / \mathrm{cm}^{2}$. Single-molecule intensity and lifetime traces were recorded for an experimental time window of $300 \mathrm{~s}$ with minimum binning times of $1 \mathrm{~ms}$ (intensity) and $100 \mathrm{~ms}\left(\tau_{f}\right)$. 

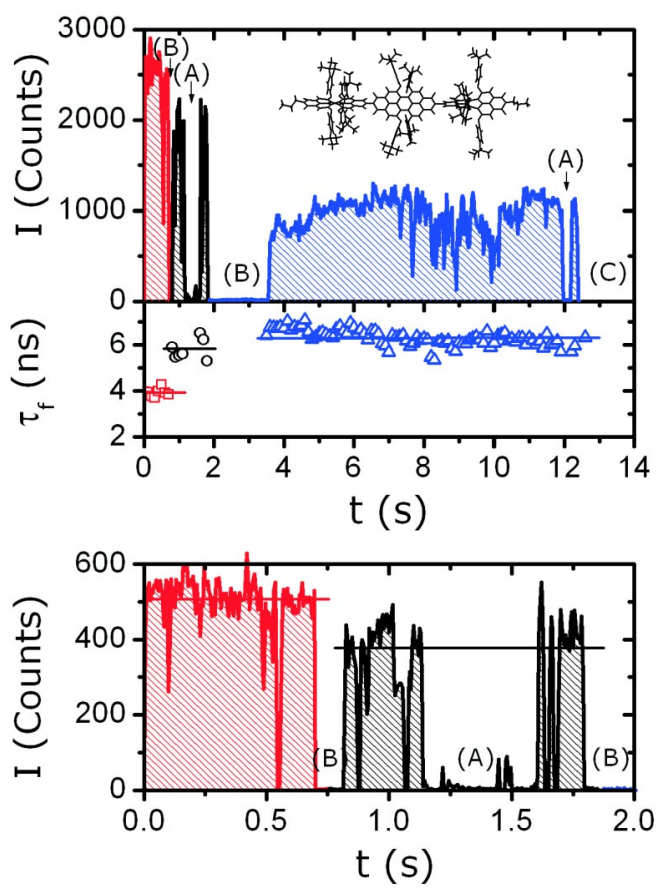

FIG. 1 (color online). Intensity $(I)$ and fluorescence lifetime $\left(\tau_{f}\right)$ trace of a single TPD molecule (molecular geometry in the inset of the top panel). The intensity trace was binned at $50 \mathrm{~ms}$, the lifetime was calculated every $100 \mathrm{~ms}$. Different levels, denoted by the different number of active chromophore units, are denoted as follows: level 3 (trimer) squares, red online $\left(\tau_{f}\right)$; level 2 (dimer), black circles; level 1 (monomer) triangles, blue online. We distinguish between three types of long-lived off states: (A) intralevel, (B) interlevel, and (C) photobleaching. Dark state (C) lasted until $t=300 \mathrm{~s}$. The lower panel gives an enlargement of the first two seconds of the trace.

The typical intensity and fluorescence lifetime trajectory for a single TPD molecule is shown in Fig. 1. Three different intensity levels can be clearly distinguished, which result from the sequential photodestruction of the PD chromophores (quantum yield $\Phi_{\mathrm{TPD}} \sim \Phi_{\mathrm{PD}}$ ). The absence of polarization changes of the fluorescence signal indicates the absence of conformational or orientational changes [18]. The intensity changes are accompanied by simultaneous changes in $\tau_{f}$, demonstrating the superradiant character of the TPD molecule [18]. After photodestruction of one PD unit, the two remaining, coupled units form a dimer, whose oscillator strength is lower than for the trimer, resulting in a lifetime increase. After the next photodegradation event, the lifetime further increases to that of the PD monomer.

Several LD states, at which the fluorescence intensity temporarily drops to the background level, can be observed in Fig. 1 [marked $(A)$ and $(B)$ ]. Such LD states were found in $93 \%$ of all 214 trimers investigated. For the trimer and dimer level this means that the active units collectively cease their emission. We attribute this collective LD state behavior to the formation of a cationic radical $\mathrm{PD}^{+}$unit. For perylene dyes, the absorption spectrum of the cationic radical is known to overlap with the emission spectrum of the neutral chromophore [19]. Moreover, the existence of a monomeric cationic radical state is possible due to the perpendicular arrangement of the three aromatic subunits in the trimer, which precludes electronic delocalization. We also find further evidence for radical formation in the study of the population and depopulation kinetics of the LD states, as described below. The electronic excitation energy of the remaining PD unit(s) is transferred to the $\mathrm{PD}^{+}$unit, which then returns to the radical ground state via a nonradiative pathway. This collectivity allows us to uniquely separate the LD states from the photobleaching events, where transitions in fluorescence intensity level and lifetime occur. Furthermore, we can distinguish between intralevel LD states [the same number of active fluorophores before and after the LD state, marked $(A)$ in Fig. 1], and interlevel LD states [a reduced number of active fluorophores after the LD state, marked $(B)]$. After successive degradation of all active PD units, the molecule is completely photobleached [interval $(C)$ ].

As observed in Fig. 1, the duration of LD states spans a broad range of values, going from $0.025 \mathrm{~s}$ to more than $1 \mathrm{~s}$. In some molecules, LD states of over $100 \mathrm{~s}$ duration were observed. These time scales are all much longer than typical triplet lifetimes [2-5].

The LD state probability density $P\left(\tau_{\text {off }}\right)$ for 199 molecules is plotted in Fig. 2(a). $P\left(\tau_{\text {off }}\right)$ is calculated from the histogram of the LD state duration by scaling each nonzero bin with the time window in which the event(s) occurred as defined by the nearest nonzero bins [9]. Interestingly, the LD state probability density does not follow a single-exponential decay. In Fig. 2(b), the same data is plotted on a log-log scale, showing a linear behavior. This corresponds to power-law dependence $\sim \tau^{m}$ with $m=1.39 \pm 0.04$.

In the case of semiconductor nanocrystal luminescence a power-law distributed blinking behavior has been observed [9-13] and ascribed to charge separation by electron tunneling [10-13]. The probability for an electron to tunnel a distance $r$ over an energy barrier of height $\Delta E$ can be approximated by $P(r) \sim \exp \left(-\kappa_{0} r\right)$, where $\kappa_{0}$ is a spatial tunneling rate given by $\kappa_{0}=2\left(2 m \Delta E / \hbar^{2}\right)^{1 / 2}$, with $m$ being the electron mass. Because of the exponential distance dependence, electron tunneling rates can vary drastically for variations in distance of only one to two nanometers. The power-law behavior then results from the summation of these widely varying time distributions [12]. However, the observed power-law behavior in our trimer system could also result from the averaging over many molecules, each with its own (single-exponential) $\tau_{\text {off }}$ distribution.

To investigate the origin of the power-law distribution, we calculated $P\left(\tau_{\text {off }}\right)$ for the sequence of LD states in $a$ single-molecule trace. The result is shown in Fig. 2(c) where a clear power-law behavior $\tau^{-m_{\text {off }}}$ with $m_{\text {off }}=$ $1.47 \pm 0.05$ over 5 orders of magnitude in time and 7 

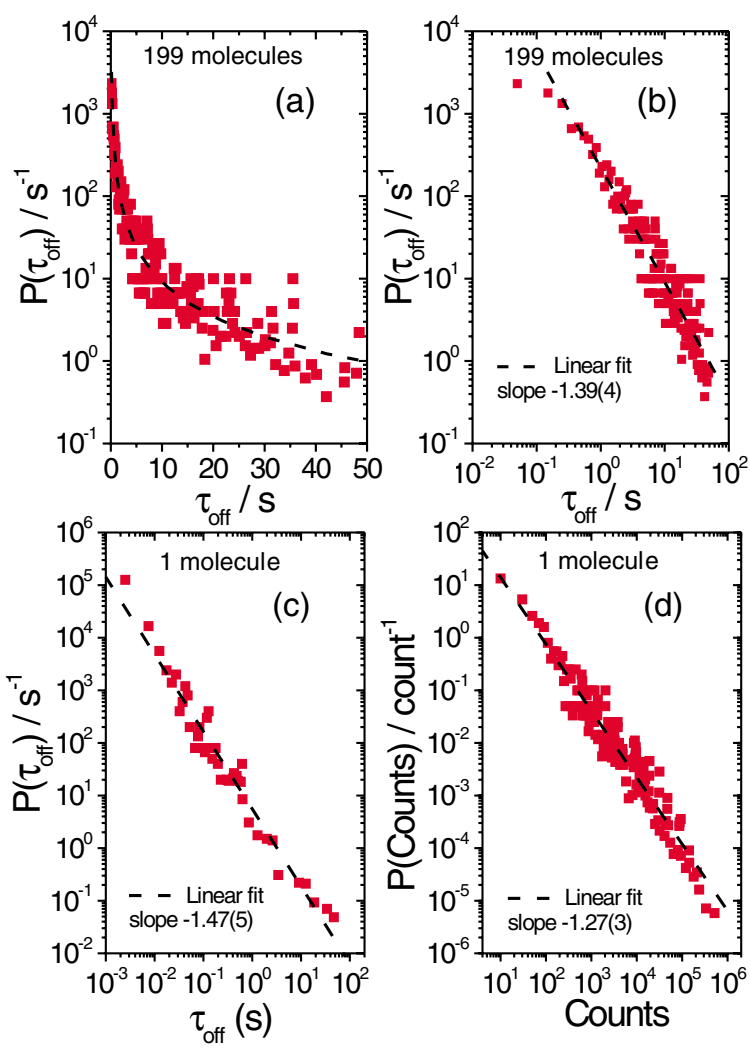

FIG. 2 (color online). Probability density for $\tau_{\text {off }}$ built (a), (b) from $1272 \mathrm{LD}$ states $\left(\tau_{\text {off }} \geq 25 \mathrm{~ms}\right)$ in 199 molecules, (a) on a log-linear scale, (b) on a log-log scale. (c) $P\left(\tau_{\text {off }}\right)$ for a single TPD molecule ( $\tau_{\text {off }} \geq 1 \mathrm{~ms}$ ). (d) Probability density for the number of photon counts before entering a LD state for the same TPD molecule. Linear fits indicate power-law behavior.

orders of magnitude in probability density is observed. Our data demonstrate for the first time that power-law distributed LD states occur in individual organic molecules. Since we have observed such behavior for different molecular environments (i.e., on bare glass) [20], we believe that power-law blinking is not a unique feature of semiconductor nanocrystals, but rather appears as a universal feature for single emitters undergoing charge separation, including fluorophores. This result is consistent with a model in which the TPD molecule is surrounded by several acceptors (or traps), each with its own back tunneling rate to the TPD molecule. A static distribution of traps then explains the observed power-law behavior of $\tau_{\text {off }}$ [12]. The electron acceptors could either be groups associated with the polymer matrix, impurities or solutes, and/or surface states at the polymer-glass interface.

Interestingly, for the same molecule, the probability density for the number of detected photon counts before entering a LD state, $P$ (counts), also exhibits power-law behavior [Fig. 2(d)]. For $P$ (counts) we find that $m_{\text {on }}=$ $1.27 \pm 0.03$, which is slightly lower than $m_{\text {off }}$. For a static distribution of acceptor states around the TPD molecule, a single rate for tunneling to any of these acceptor states would result, which would give single-exponential in- stead of power-law statistics. The power-law behavior of $P$ (counts) reflects the dynamic nature of the polymer environment. Conformational changes in the polymer matrix, taking place on the tens of milliseconds time scale [21], lead to slight, nanometer-scale, positional variations of the acceptor states distribution. This, in turn, leads to drastic variations in the average tunneling rate given the exponential distance dependence of the tunneling process.

The probability densities $P\left(\tau_{\text {off }}\right)$ and $P$ (counts) were found not to depend on the level (trimer, dimer, or monomer) in which the LD state occurred nor on the strength of the excitonic coupling, which varies from molecule to molecule $[18,22]$. A power-law behavior was also obtained on single perylene molecules under continuous-wave illumination.

Having established the population kinetics and corresponding pathway for the LD states, we investigated the relation between the LD states and photobleaching. Hereto, we exploit the excitonic coupling between the PD units in the TPD molecule and separate the LD states intralevel or interlevel (see Fig. 1). The distribution of the order of magnitude of the LD state duration for intralevel and interlevel LD states is shown in Fig. 3. As observed, interlevel LD states last markedly longer (on average a factor 3) than intralevel LD states. This highlights an intrinsic relationship between the LD states and photodegradation: the longer a molecule resides in the LD state (i.e., remains radicalized), the higher the probability that the molecule will degrade into a nonfluorescent species. Although seemingly trivial, this relationship has, to our knowledge, never been brought to evidence.

The analysis of photobleaching intermediates obeying power-law statistics may be further complicated by the presence of $\mathrm{O}_{2}$. In fact, Zondervan et al. have recently found that the presence of $\mathrm{O}_{2}$ not only enhances photo-

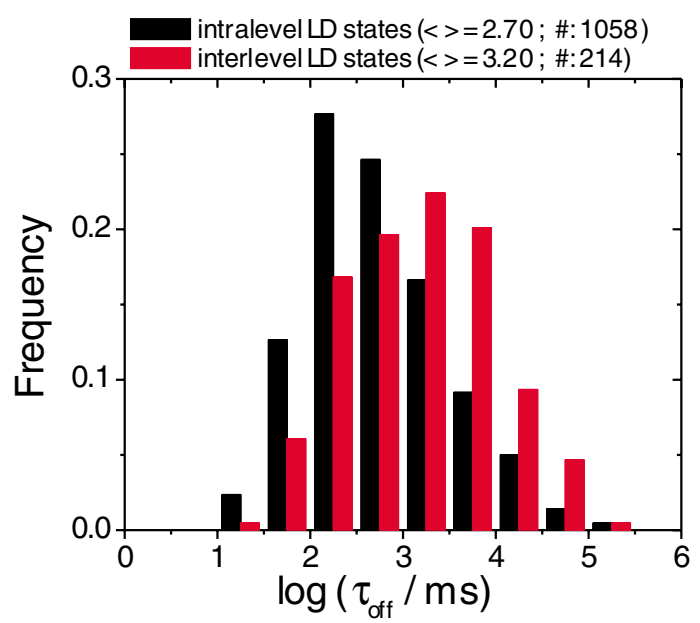

FIG. 3 (color online). Order of magnitude of LD state duration for intralevel versus interlevel LD states for 199 molecules (minimum $\tau_{\text {off }}=25 \mathrm{~ms}$ ). The average order of magnitude and the number of events in the respective distributions are indicated in brackets. 
bleaching but also assists in repopulation of the ground state from the dark state [16]. Interestingly, $\mathrm{Ha}$ and $\mathrm{Xu}$ reported the existence of multiple photobleaching intermediates, each with single-exponential kinetics, in a liquid environment, based on the observation of a complex $\mathrm{O}_{2}$-dependent behavior of the distribution of dark states [17]. It would be interesting to investigate whether a single intermediate with complicated $\mathrm{O}_{2}$-dependent power-law behavior might provide an alternative explanation for the observations of $\mathrm{Ha}$ and $\mathrm{Xu}$ [17]. Further research in this area should clarify the precise influence of $\mathrm{O}_{2}$ on the observed power-law population kinetics of the radical dark state.

Given the above relationship between LD states and photodegradation, our system allows us to quantify this coupling by directly analyzing the photodegradation events (i.e., level 3 to 2 and level 2 to 1 transitions). For the 134 transitions from level 3 to 2, 54\% were preceded by a LD state ( $\left.\tau_{\text {off }}>25 \mathrm{~ms}\right)$, for the 136 transitions from level 2 to $151 \%$, and for the 62 transitions from level 2 to 1 in a dimer system (DPD), we found $62 \%$. Thus, over $50 \%$ of the total number of photodegradation events is preceded by LD states. Moreover, given the underlying power-law distribution (Fig. 2), the number of LD states with $1 \mathrm{~ms} \leq$ $\tau_{\text {off }} \leq 25 \mathrm{~ms}$ is estimated to be 2.5 times larger than those within our experimental time window $\left(25 \mathrm{~ms} \leq \tau_{\text {off }} \leq\right.$ $300 \mathrm{~ms}$ ). Thus, although multiple photobleaching pathways and photoproducts may exist [7,17], we conclude that radical formation by electron tunneling from the fluorophore's excited state to an acceptor level in the molecule's nanoenvironment forms the dominant process for molecular photobleaching.

It is generally believed that intersystem crossing to the triplet excited state forms a precursor for molecular photobleaching. It has been noted by Zondervan et al. that dark states other than the triplet, like the radical state, can drastically affect photobleaching [16], as now strongly and directly evidenced by the results above. Using single-molecule fluorescence measurements, we cannot distinguish whether electron tunneling takes place from the singlet excited state or from the triplet state [14]. Further experiments, e.g., exploiting the heavy-atom effect to increase population of the triplet state [3], are needed to resolve this issue.

Finally, we want to note that interlevel LD states (i.e., with a subsequent photodegradation event) occurring in the TPD trimer level on average have a shorter duration than those occurring in the TPD dimer level (data not shown). This indicates that the photobleaching reacting of the radical is a process that is enhanced by the photo-induced excitation of the radical due to energy transfer from the remaining PD units [16], substantiating the fact that the collectivity of the LD states is caused by energy transfer to the radical unit.

In conclusion, exploiting the strong coupling in a superradiant trimer to distinguish radical formation leading to collective dark states from photobleaching, we have dem- onstrated that radicalization forms the main pathway to molecular photobleaching. The population and depopulation kinetics of radical dark states in a single molecule follow power-law statistics, indicating electron tunneling to a time-varying distribution of acceptor states in the polymer matrix. Thus, power-law distributed blinking, previously reported in the case of semiconductor nanocrystals, appears as a universal feature of single emitters undergoing charge separation. Prevention of electron tunneling, e.g., by modification of the chemical structure of either fluorophore or the fluorophore environment, may reduce both photoblinking and photobleaching. This finding may lead to highly photostable fluorophores, crucial for single-molecular photonics. Furthermore, the chemical and physical tunability of single molecules and their environment opens ways to further explore the details of the electron tunneling process. These and further experiments will furthermore help clarifying the different existing models for electron tunneling and blinking in semiconductor nanocrystals [10-13].

We are grateful to J. J. García-López, D. N. Reinhoudt, and M. Crego-Calama for providing the TPD molecules. We also thank L. Kuipers for fruitful discussions. This work has been supported by the Volkswagen Stiftung (J.P. H.), the Stichting voor Fundamenteel Onderzoek der Materie (E. M. P. H. v. D.), and a Marie Curie Fellowship in EC Program No. IHP-99 (J. H.).

*E-mail: j.p.hoogenboom@utwente.nl

[1] R. J. Cook and H. J. Kimble, Phys. Rev. Lett. 54, 1023 (1985).

[2] J. A. Veerman et al., Phys. Rev. Lett. 83, 2155 (1999).

[3] F. Kohn et al., J. Phys. Chem. A 106, 4808 (2002).

[4] M. Haase et al., J. Phys. Chem. B 108, 10445 (2004).

[5] P. Tinnefeld et al., Chem. Phys. Chem. 5, 1786 (2004).

[6] R. M. Dickson et al., Nature (London) 388, 355 (1997).

[7] T. Christ et al., Angew. Chem., Int. Ed. 40, 4192 (2001).

[8] J. Hernando et al., J. Phys. Chem. A 107, 43 (2003).

[9] M. Kuno et al., J. Chem. Phys. 115, 1028 (2001).

[10] K. T. Shimizu et al., Phys. Rev. B 63, 205316 (2001).

[11] R. Verberk, A. M. van Oijen, and M. Orrit, Phys. Rev. B 66, 233202 (2002).

[12] M. Kuno et al., Phys. Rev. B 67, 125304 (2003).

[13] A. Issac, C. von Borczyskowski, and F. Cichos, Phys. Rev. B 71, 161302 (2005).

[14] R. Zondervan et al., J. Phys. Chem. A 107, 6770 (2003).

[15] J. Yu, D. H. Hu, and P.F. Barbara, Science 289, 1327 (2000).

[16] R. Zondervan et al., J. Phys. Chem. A 108, 1657 (2004).

[17] T. Ha and J. Xu, Phys. Rev. Lett. 90, 223002 (2003).

[18] J. Hernando et al., Phys. Rev. Lett. 93, 236404 (2004).

[19] T. Kircher and H.-G. Löhmannsröben, Phys. Chem. Chem. Phys. 1, 3987 (1999).

[20] J.P. Hoogenboom et al. (to be published).

[21] R. A. L. Vallée et al., Phys. Rev. Lett. 91, 038301 (2003).

[22] M. Lippitz et al., Phys. Rev. Lett. 92, 103001 (2004). 